\title{
A Note on the Periodicity of the Lyness Max Equation
}

\author{
Ali Gelisken, Cengiz Cinar, and Ramazan Karatas \\ Department of Mathematics, Faculty of Education, Selcuk University, 42099 Konya, Turkey
}

Correspondence should be addressed to Cengiz Cinar, ccinar25@yahoo.com

Received 24 October 2007; Accepted 21 December 2007

Recommended by Elena Braverman

We investigate the periodic nature of solutions of a "max-type" difference equation sometimes referred to as the "Lyness max" equation. The equation we consider is $x_{n+1}=\max \left\{x_{n}, A\right\} / x_{n-1}$. where $A$ is a positive real parameter, $x-1=A^{r_{-1}}$, and $x_{0}=A^{r_{0}}$ such that $r_{-1}$ and $r_{0}$ are positive rational numbers. The results in this paper answer the Open Problem of Grove and Ladas (2005).

Copyright (c) 2008 Ali Gelisken et al. This is an open access article distributed under the Creative Commons Attribution License, which permits unrestricted use, distribution, and reproduction in any medium, provided the original work is properly cited.

\section{Introduction}

In [1], the following open problem was posed.

Open problem 1. Assume that $A \in(0, \infty)$, and that $r_{1}$ and $r_{2}$ are positive rational numbers. Investigate the periodic nature of the solution of the difference equation

$$
x_{n+1}=\frac{\max \left\{x_{n}, A\right\}}{x_{n-1}}, \quad n=0,1, \ldots
$$

with initial conditions $x_{-1}=A^{r_{1}}$ and $x_{0}=A^{r_{2}}$.

In [2], Janowski et al. proved the following result.

Theorem 1.1. The solution of (1.1) with initial conditions $x_{-1}=1, x_{0}=A^{k / m}$, where $(k, m)=1$ and $1 \leq m<k$, is periodic

(1) with period $5 k-m$ if $A>1$,

(2) with period $5 k+m$ if $A<1$. 
In [3], Feuer proved the following results.

Theorem 1.2. Assume that $A>1$ and that $\left\{x_{n}\right\}$ is a solution of (1.1). If $\left\{x_{n}\right\}$ is periodic of period $p$, then $p=5 r+4 s$ for some positive integers $r$ and $s$. In fact, there exist positive integers $r_{1}$ and $s_{1}$ such that $\left\{x_{n}\right\}$ is periodic of prime period $5 r_{1}+4 s_{1}$.

Lemma 1.3. Assume that $A>1$ and that $\left\{x_{n}\right\}$ is a solution of (1.1). If $\left\{x_{n}\right\}$ is a periodic solution of prime period $p=5 r+4 s$, where $(r, s)=1$ and $A \leq x_{0}<x_{-1}$, then

$$
x_{-1}=A^{r / s+1} \text {. }
$$

Theorem 1.4. Assume that $A<1$ and that $\left\{x_{n}\right\}$ is a solution of (1.1). If $\left\{x_{n}\right\}$ is periodic of period $p$, then $p=5 r+6 s$ for some positive integers $r$ and $s$. In fact, there exist positive integers $r_{1}$ and $s_{1}$ such that $\left\{x_{n}\right\}$ is periodic of prime period $5 r_{1}+6 s_{1}$.

Lemma 1.5. Assume that $A<1$ and that $\left\{x_{n}\right\}$ is a solution of (1.1). If $\left\{x_{n}\right\}$ is a periodic solution of prime period $p=5 r+6 s$, where $(r, s)=1$ and $x_{0} \leq A<1<x_{-1}$, then

$$
\frac{x_{0}}{x_{-1}}=A^{r / s+1}
$$

In [2], a smaller range of solutions was presented with $x_{0}=A$ in the case $A>1$ (and similar results for $A<1$ ). In [3], it was shown that any solution with initial conditions $1<A \leq$ $x_{0}<x_{-1}=A^{r / s+1}$ is periodic with period $5 r+4 s$ (and similar results for $A<1$ ). So, not all possible periods were discovered in $[2,3]$. We solve the open problem exactly.

In [4], it was shown that every solution of

$$
a_{n+2}=\max \left\{a_{n+1}, 0\right\}-a_{n}, \quad n=0,1, \ldots,
$$

is periodic with period 5 . The change of variables

$$
x_{n-1}=e^{a_{n}} \text { for } n \geq 0
$$

reduces (1.4) to (1.1) with $A=1$.

\section{The case $A>1$}

We consider (1.1). Let $x_{n}=A^{r_{n}}$ for $n \geq-1$. Then, (1.1) implies the difference equation

$$
r_{n+1}=\max \left\{r_{n}, 1\right\}-r_{n-1}, \quad n=0,1, \ldots,
$$

where initial conditions $r_{-1}$ and $r_{0}$ are positive rational numbers.

We give the following three lemmas which give us explicit solutions for some consecutive terms and show us the pattern of the behavior of solutions.

Lemma 2.1. Let $r_{n}$ be a solution of (2.1) such that $\max \left\{r_{-1}, r_{0}\right\}=r>1$. Then the following statements are true for some integers $N \geq-1$. 
(1) If $r_{N}=r, r_{N}=r_{N+4}$, or $r_{N}=r_{N+5}$.

(2) If $r_{N}=r_{N+4}=r, r_{N+1} \leq 1$, and $r_{N+5}=r_{N+1}+r_{N}-1$.

(3) If $r_{N}=r_{N+5}=r, r_{N+1}>1$, and $r_{N+6}=r_{N+1}-1$.

Proof. (1) Let $r_{N}=r$ for some integers $N \geq-1$. By computer computation, we have $r_{N-1}<r_{N}$. From (2.1), we get that $r_{N+1}=r_{N}-r_{N-1}$.

If $r_{N+1} \leq 1$, we get

$$
r_{N+2}=1-r_{N}<0, \quad r_{N+3}=1-r_{N+1}<1, \quad r_{N+4}=r_{N}
$$

If $r_{N+1}>1$, we get

$$
r_{N+2}=-r_{N-1}<0, \quad r_{N+3}=1-r_{N+1}<1, \quad r_{N+4}=1+r_{N-1}>1, \quad r_{N+5}=r_{N} .
$$

(2)-(3). From (1) and (2.1), we get immediately (2) and (3).

Clearly, there are infinite $N$ integers which hold Lemma 2.1. The proof of the following lemma about a number of $N$ integers is by induction and will be omitted.

Lemma 2.2. Let $r_{n}$ be a solution of (2.1) which holds Lemma 2.1. Let $r=k / m,(k, m)=1$, and $N<5 k-m-1$. Then, a number of $N$ integers

(1) which hold 2.1(1) is $k$.

(2) which hold 2.1(2) is $m$.

(3) which hold 2.1(3) is $k-m$.

Remark 2.3. Apply Lemma 2.1. Firstly, from Lemma 2.1(2), we get that

$$
r_{N}=r_{N+4}=r, \quad r_{N+5}=r_{N+1}+r_{N}-1 .
$$

Then, from Lemma 2.1(3), we get

$$
r_{N+9}=r_{N+4}=r, \quad r_{N+10}=r_{N}+r_{N+1}-2 .
$$

Now, we apply Lemma 2.1(3) firstly and then we get that

$$
r_{N}=r_{N+5}=r, \quad r_{N+6}=r_{N+1}-1 \text {. }
$$

Then from Lemma 2.1(2), we get

$$
r_{N+9}=r_{N+5}=r, \quad r_{N+10}=r_{N}+r_{N+1}-2 .
$$

It shows that the last corresponding two terms are same in each two cases. So, we can apply Lemmas 2.1(2) or 2.1(3) firstly for getting the last two terms we need.

We give the following lemma which is taken from Lemmas 2.1, 2.2, and Remark 2.3. It allows us to calculate more quickly terms in the solution. 
Lemma 2.4. Suppose that $r_{n}$ satisfies Lemma 2.1 with $r=k / m,(k, m)=1$. Then the following hold.

(1) If $r_{N}=r_{N+4 l_{1}}=r$, then $r_{N+4 l_{1}+1}=r_{N}+r_{N+4\left(l_{1}-1\right)+1}-1$ for $l_{1}=1,2, \ldots, m$.

(2) If $r_{N+4 m}=r_{N+4 m+5 l_{2}}=r$, then $r_{N+4 m+5 l_{2}+1}=r_{N+4 m+5\left(l_{2}-1\right)+1}-1$ for $l_{2}=1,2, \ldots, k-m$.

Theorem 2.5. Let $r_{n}$ be a solution of (2.1), where $r_{-1}, r_{0}$ are positive rational numbers. Suppose that $\max \left\{r_{-1}, r_{0}, 1\right\}=k / m$ and $(k, m)=1$. Then, $r_{n}$ is periodic with prime period $5 k-m$.

Proof. We have to show $r_{n}=r_{n+5 k-m}$ for all $n \geq-1$. We have $r \geq 1$.

If $k / m=1$, we have $k=m=1,0<r_{-1} \leq 1$, and $0<r_{0} \leq 1$. Then, from (2.1) we get that

$$
r_{1}=1-r_{-1}, \quad r_{2}=1-r_{0}, \quad r_{3}=r_{-1}, \quad r_{4}=r_{0}
$$

So, the proof is completed for $r=1$.

We assume that $k / m>1$. We will apply Lemma 2.4. From Lemma 2.4(1), we get that

$$
r_{N}=r_{N+4 m}, \quad r_{N+4 m+1}=m r_{N}+r_{N+1}-m .
$$

Then, from Lemma 2.4(2) we get

$$
r_{N+4 m}=r_{N+4 m+5(k-m)}, \quad r_{N+4 m+5(k-m)+1}=m r_{N}+r_{N+1}-k .
$$

So, at the end of this process we have $r_{N}=r_{N+5 k-m}$ and $r_{N+1}=r_{N+5 k-m+1}$. From $r_{n-1}=$ $\max \left\{r_{n}, 1\right\}-r_{n+1}$, we get immediately $r_{N}=r_{N+5 k-m}$ for all $N \geq-1$. Also, it is easy to see that $r_{N+1} \neq r_{N+4 l_{1}+1}$ and $r_{N+1} \neq r_{N+4 m+5 l_{2}+1}$ for $l_{1}=1,2, \ldots, m$ and $l_{2}=1,2, \ldots, k-m-1$. It shows that $5 k-m$ is the smallest period. So, the proof is completed.

\section{The Case $A<1$}

We consider (1.1). Let $x_{n}=A^{r_{n}}$ for $n \geq-1$. Then (1.1) implies the difference equation

$$
r_{n+1}=\min \left\{r_{n}, 1\right\}-r_{n-1}, \quad n=0,1, \ldots,
$$

where initial conditions are positive rational numbers.

The proofs of the lemmas and theorems in this section are similar to the proofs of the corresponding lemmas and theorems in the previous section and will be omitted.

Lemma 3.1. Let $r_{n}$ be a solution of (3.1) such that at least one of the initial conditions is $0<r_{-1} \leq 1$ or $0<r_{0} \leq 1$. Let $\max \left\{r_{-1}, r_{0}\right\}=r$. Then the following statements are true for some integers $N>0$.

(1) If $r_{N}=-r$, then $r_{N}=r_{N+5}$ or $r_{N}=r_{N+6}$.

(2) If $r_{N}=r_{N+6}=-r$, then $r_{N+5}=r_{N-1}+r_{N}+1$.

(3) If $r_{N}=r_{N+5}=-r$, then $r_{N+4}=r_{N-1}+1$.

The following lemma is generalized from Lemma 3.1.

Lemma 3.2. Let $r_{n}$ be a solution of (3.1) which holds Lemma 3.1. Let $r=k / m$ and $(k, m)=1$. Then, the following hold. 
(1) If $r_{N}=r_{N+6 l_{1}}=-r$, then $r_{N+6 l_{1}-1}=r_{N}+r_{N+6\left(l_{1}-1\right)-1}+1$ for $l_{1}=1,2, \ldots, m$.

(2) If $r_{N+6 m}=r_{N+6 m+5 l_{2}}=-r$, then $r_{N+6 m+5 l_{2}-1}=r_{N+6 m+5\left(l_{2}-1\right)-1}+1$ for $l_{2}=1,2, \ldots, k-m$.

The following result follows directly from previous lemma. We assume that at least one of the initial conditions $r_{-1}, r_{0}$ is $0<r_{-1} \leq 1$ or $0<r_{0} \leq 1$. We will demonstrate what prime period for a solution of (3.1) in these conditions.

Theorem 3.3. Let $r_{n}$ be a solution of (3.1). Suppose that $\max \left\{r_{-1}, r_{0}, 1\right\}=k / m$ and $(k, m)=1$. Then, $r_{n}$ is periodic with prime period $5 k+m$.

Lemma 3.4. Let $r_{n}$ be a solution of (3.1), where $r_{-1}>1$ and $r_{0}>1$. Let $r_{-1}+r_{0}=r$. Then the following statements are true for some integers $N>0$.

(1) If $r_{N}=1-r$, then $r_{N}=r_{N+5}$ or $r_{N}=r_{N+6}$.

(2) If $r_{N}=r_{N+6}=1-r$, then $r_{N+5}=r_{N-1}+r_{N}+1$.

(3) If $r_{N}=r_{N+5}=1-r$, then $r_{N+4}=r_{N-1}+1$.

Lemma 3.5. Let $r_{n}$ be a solution of (3.1) which holds Lemma 3.4. Let $r=k / m$ and $(k, m)=1$. Then, the following hold.

(1) If $r_{N}=r_{N+6 l_{1}}=1-r$, then $r_{N+6 l_{1}-1}=r_{N}+r_{N+6\left(l_{1}-1\right)-1}+1$ for $l_{1}=1,2, \ldots, m$.

(2) If $r_{N+6 m}=r_{N+6 m+5 l_{2}}=1-r$, then $r_{N+6 m+5 l_{2}-1}=r_{N+6 m+5\left(l_{2}-1\right)-1}+1$ for $l_{2}=1,2, \ldots, k-2 m$.

Theorem 3.6. Let $r_{n}$ be a solution of (3.1), where $r_{-1}>1$ and $r_{0}>1$. Let $r_{-1}+r_{0}=r, r=k / m$, and $(k, m)=1$. Then, $r_{n}$ is periodic with prime period $5 k-4 m$.

\section{References}

[1] E. A. Grove and G. Ladas, Periodicities in Nonlinear Difference Equations, vol. 4 of Advances in Discrete Mathematics and Applications, Chapman \& Hall/CRC, Boca Raton, Fla, USA, 2005.

[2] E. J. Janowski, V. L. Kocic, G. Ladas, and S. W. Schultz, "Global behavior of solutions of $x_{n}+1=$ $\max \left\{x_{n}, A\right\} / x_{n-1}, "$ in Proceedings of the 1st International Conference on Difference Equations, pp. 273-282, Gordon and Breach, San Antonio, Tex, USA, 1994.

[3] J. Feuer, "Periodic solutions of the Lyness max equation," Journal of Mathematical Analysis and Applications, vol. 288, no. 1, pp. 147-160, 2003.

[4] Problem E3437 [1991,366], American Mathematical Monthly, November 1992. 